\title{
Acoustic wavefield separation using horizontal receiver arrays deployed at multiple depths on land
}

Joost van der Neut* , Delft University of Technology; Andrey Bakulin, Saudi Aramco, EXPEC Advanced Research Center, and Dmitry Alexandrov, St. Petersburg State University

\section{SUMMARY}

We present a novel inversion scheme for decomposing upgoing and downgoing wavefields from vertical particle velocity recordings in downhole arrays at multiple depth levels. Our method requires no knowledge of the subsurface medium parameters as the required operators are obtained directly from the data by direct-wave interferometry. As we demonstrate, the method can be applied with as few as two receiver arrays, as long as their vertical spacing is sufficiently small. Additional depth levels can be used to improve the stability of the inversion.

\section{INTRODUCTION}

It is well known that upgoing and downgoing wavefields can be separated by combining measurements of pressure and particle velocity (Claerbout, 1971). This concept has been applied to multi-component ocean-bottom cable data (Schalkwijk et al., 1999), dual-sensor streamer data (Kluver et al., 2009) and from dual-sensor measurements in horizontal boreholes (Mehta et al., 2010). An alternative strategy for up / down separation is to combine measurements from different depths. This method has been applied to streamer data in socalled over / under acquisition (Posthumus, 1993) as well as in vertical boreholes (Loewenthal and Robinson, 2000; Petronio and Poletto, 2010). We propose to apply up / down separation using measurements in two or more horizontal receiver arrays at different depth levels. Such a configuration with sensors at multiple depth levels has been tested for $\mathrm{CO}_{2}$ monitoring (Bakulin et al., 2012), where wavefield separation was applied to obtain time-lapse images without and with virtual source redatuming (Bakulin and Calvert, 2006). Up / down separation using hydrophone-geophone summation has proven to be useful for general processing of buried array data (Burnstad et al., 2012a), for the virtual source method (Mehta et al., 2007) and related technologies such as interferometry by multidimensional deconvolution (Wapenaar et al., 2011). Subsequently, hydrophone data on land may be of variable quality and repeatability (Burnstad et al., 2012b) and therefore an alternative wavefield separation technique using geophone sensors only can be very relevant. Such method is particularly attractive for deployments using shallow vertical holes, which allow installation of vertical arrays.

We consider the configuration as shown in Figure 1. Vertical geophones are deployed in arrays A and B, located at depth levels $z_{A}$ and $z_{B}$, respectively. In the field data example from Saudi Arabia (Bakulin et al., 2012) sensors were deployed at multiple levels in shallow vertical holes. The sources are located at the surface above the receivers. To separate the wavefield into upgoing and downgoing components, the measurements of both arrays should be combined. For this purpose, we use wavefield extrapolation techniques that can redatum a wavefield from one level to another. In the next section we will briefly review these techniques and the required propagators. Thereafter we show how these propagators can be estimated using direct-wave interferometry. Then we will introduce a novel wavefield separation scheme and illustrate it with an example. Finally, we will discuss some practical aspects regarding the spacing between the receiver arrays and the potential benefits of using more than two arrays for wavefield separation.

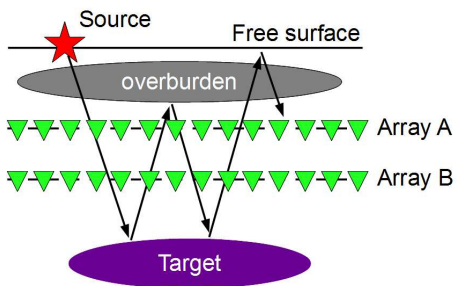

Figure 1: Configuration for wavefield separation with two levels of sensors: array A and array B.

\section{REVIEW OF WAVEFIELD EXTRAPOLATION}

We introduce $\hat{V}^{+}\left(\mathbf{x}_{A}, \mathbf{x}_{S} ; \omega\right)$ as the downgoing particle velocity field recorded at array A (at level $z_{A}$ ), given in the frequencyspace domain (indicated by the caret), where $\omega$ is the angular frequency, $\mathbf{x}_{A}$ is the receiver location and $\mathbf{x}_{S}$ is the source location. This field can be propagated to level $z_{B}$ by forward wavefield extrapolation:

$$
\hat{V}^{+}\left(\mathbf{x}_{B}, \mathbf{x}_{S} ; \omega\right)=\int_{\Omega_{A}} \hat{W}^{+}\left(\mathbf{x}_{B}, \mathbf{x}_{A} ; \omega\right) \hat{V}^{+}\left(\mathbf{x}_{A}, \mathbf{x}_{S} ; \omega\right) d \mathbf{x}_{A}
$$

The integral is carried out over a plane of horizontal coordinates $\Omega_{A}$. In theory, $\Omega_{A}$ should range from $-\infty$ to $+\infty$, but it can be limited to a small aperture as we will see later. $\hat{W}^{+}\left(\mathbf{x}_{B}, \mathbf{x}_{A} ; \omega\right)$ is a particle velocity normalized propagator:

$$
\hat{W}^{+}\left(\mathbf{x}_{B}, \mathbf{x}_{A} ; \omega\right)=2 \rho\left(\mathbf{x}_{B}\right) \frac{\partial \hat{G}\left(\mathbf{x}_{B}, \mathbf{x}_{A} ; \omega\right)}{\partial z_{B}}
$$

This expression can be derived in a similar way as the expressions by Wapenaar (1998), who defined propagators with pressure and power-flux normalization. In equation $2, \rho\left(\mathbf{x}_{B}\right)$ is the density at $\mathbf{x}_{B}$. Further, $\hat{G}\left(\mathbf{x}_{B}, \mathbf{x}_{A} ; \omega\right)$ is a Green's functions with a source at $\mathbf{x}_{A}$ and a receiver at $\mathbf{x}_{B}$ in a reference medium, which is vertically shift invariant for $z<z_{A}$ and $z>z_{B}$. This medium can be smoothly varying between levels $z_{A}$ and $z_{B}$. 


\section{Wavefield separation using sensors at multiple depths on land}

In a similar way, the upgoing field $\hat{V}^{-}\left(\mathbf{x}_{A}, \mathbf{x}_{S} ; \omega\right)$ can be datumed to level $z_{B}$ by inverse wavefield extrapolation:

$$
\hat{V}^{-}\left(\mathbf{x}_{B}, \mathbf{x}_{S} ; \omega\right)=\int_{\Omega_{A}} \hat{F}^{-}\left(\mathbf{x}_{B}, \mathbf{x}_{A} ; \omega\right) \hat{V}^{-}\left(\mathbf{x}_{A}, \mathbf{x}_{S} ; \omega\right) d \mathbf{x}_{A} .
$$

The required propagator $\hat{F}^{-}\left(\mathbf{x}_{B}, \mathbf{x}_{A} ; \omega\right)$ for this process is closely related to the forward propagator since (Wapenaar, 1998):

$$
\hat{F}^{-}\left(\mathbf{x}_{B}, \mathbf{x}_{A} ; \omega\right) \approx\left(\hat{W}^{+}\left(\mathbf{x}_{B}, \mathbf{x}_{A} ; \omega\right)\right)^{*}
$$

where $*$ denotes complex conjugation. The approximation sign is applied here, because equation 4 is not valid for the evanescent wavefield, which will be neglected in this analysis. To apply forward propagation of the downgoing field and inverse propagation of the upgoing field, we require knowledge of the propagator $\hat{W}^{+}\left(\mathbf{x}_{B}, \mathbf{x}_{A} ; \omega\right)$ and its complex conjugate. In the following section, we show how we can estimate this propagator with direct-field interferometry.

\section{INTERFEROMETRIC PROPAGATOR ESTIMATION}

We assume that the direct (indicated by superscript 0 ) fields $\hat{V}^{0}\left(\mathbf{x}_{A}, \mathbf{x}_{S} ; \omega\right)$ at level $z_{A}$ and $\hat{V}^{0}\left(\mathbf{x}_{B}, \mathbf{x}_{S} ; \omega\right)$ at level $z_{B}$ can be isolated using a time-gate (Bakulin and Calvert, 2006). We assume that these fields are purely downgoing, such that the following formulation can be derived at each depth level, similar to equation 1 :

$$
\hat{V}^{0}\left(\mathbf{x}_{B}, \mathbf{x}_{S} ; \omega\right)=\int_{\Omega_{A}} \hat{W}^{+}\left(\mathbf{x}_{B}, \mathbf{x}_{A} ; \omega\right) \hat{V}^{0}\left(\mathbf{x}_{A}, \mathbf{x}_{S} ; \omega\right) d \mathbf{x}_{A} .
$$

Although equation 5 is still valid if the medium between levels $z_{A}$ and $z_{B}$ is inhomogeneous, there is a strict assumption that $\hat{V}^{0}$ should be downgoing at each depth level. Any upgoing components in this field will not be handled correctly. We introduce the normal equation of inverse problem 5, following Wapenaar et al. (2011):

$$
\hat{C}\left(\mathbf{x}_{B}, \mathbf{x}_{A}^{\prime} ; \omega\right)=\int_{\Omega_{A}} \hat{W}^{+}\left(\mathbf{x}_{B}, \mathbf{x}_{A} ; \omega\right) \hat{\Gamma}\left(\mathbf{x}_{A}, \mathbf{x}_{A}^{\prime} ; \omega\right) d \mathbf{x}_{A}
$$

where $\mathbf{x}_{A}^{\prime}$ is a location in array A, $\hat{C}$ is the correlation function

$$
\hat{C}\left(\mathbf{x}_{B}, \mathbf{x}_{A}^{\prime} ; \omega\right)=\sum_{n} \hat{V}^{0}\left(\mathbf{x}_{B}, \mathbf{x}_{S}^{(n)} ; \omega\right)\left\{\hat{V}^{0}\left(\mathbf{x}_{A}^{\prime}, \mathbf{x}_{S}^{(n)} ; \omega\right)\right\}^{*}
$$

$n$ is the source index and $\hat{\Gamma}$ is the point-spread function:

$$
\hat{\Gamma}\left(\mathbf{x}_{A}, \mathbf{x}_{A}^{\prime} ; \omega\right)=\sum_{n} \hat{V}^{0}\left(\mathbf{x}_{A}, \mathbf{x}_{S}^{(n)} ; \omega\right)\left\{\hat{V}^{0}\left(\mathbf{x}_{A}^{\prime}, \mathbf{x}_{S}^{(n)} ; \omega\right)\right\}^{*}
$$

In theory, the propagator can be retrieved by straightforward inversion of the normal equation. However, this will only be successful if the time-gates are accurately chosen. Instead, we prefer to constrain the inversion process by allowing the unknown operator $\hat{W}^{+}\left(\mathbf{x}_{B}, \mathbf{x}_{A}, \omega\right)$ to have only one non-zero element per trace. In this way the number of unknown parameters are severely reduced, whereas the operator can still shift and rescale data as it should to obey equation 6 . This condition is imposed by writing the propagator in the following way:

$$
\hat{W}^{+}\left(\mathbf{x}_{B}, \mathbf{x}_{A} ; \omega\right) \approx A_{W}\left(\mathbf{x}_{B}, \mathbf{x}_{A}\right) \exp \left(-j \omega \Delta t_{W}\left(\mathbf{x}_{B}, \mathbf{x}_{A}\right)\right) .
$$

Here $A_{W}\left(\mathbf{x}_{B}, \mathbf{x}_{A}\right)$ is a frequency-independent amplitude scaling factor and $\Delta t_{W}\left(\mathbf{x}_{B}, \mathbf{x}_{A}\right)$ is a frequency-independent time shift. From the theory of the virtual source method and seismic interferometry (Bakulin and Calvert, 2006) we know that the correlation function provides a good estimate of the propagator's traveltime. Therefore we pick the largest peak in each trace of $\hat{C}\left(\mathbf{x}_{B}, \mathbf{x}_{A}^{\prime} ; \omega\right)$ and assign it to $\Delta t_{W}\left(\mathbf{x}_{B}, \mathbf{x}_{A}\right)$ at $\mathbf{x}_{A}^{\prime}=\mathbf{x}_{A}$. These time delays are used to propagate the point-spread functions kinematically from level $z_{A}$ to level $z_{B}$, according to

$\hat{\Gamma}_{\text {shifted }}\left(\mathbf{x}_{B}, \mathbf{x}_{A}, \mathbf{x}_{A}^{\prime} ; \omega\right)=\exp -\left(j \omega \Delta t_{W}\left(\mathbf{x}_{B}, \mathbf{x}_{A}\right)\right) \hat{\Gamma}\left(\mathbf{x}_{A}, \mathbf{x}_{A}^{\prime} ; \omega\right)$

By substituting equation 9 into equation 6 and transforming to the time-domain, we arrive at

$$
C\left(\mathbf{x}_{B}, \mathbf{x}_{A}^{\prime} ; t\right)=\int_{\Omega_{A}} A_{W}\left(\mathbf{x}_{B}, \mathbf{x}_{A}\right) \Gamma_{\text {shifted }}\left(\mathbf{x}_{B}, \mathbf{x}_{A}, \mathbf{x}_{A}^{\prime} ; t\right) d \mathbf{x}_{A}
$$

Here, $\Gamma_{\text {shifted }}\left(\mathbf{x}_{B}, \mathbf{x}_{A}, \mathbf{x}_{A}^{\prime} ; t\right)$ is the point-spread function after kinematic shifting as defined by equation 10 . Equation 11 can be inverted for $A_{W}\left(\mathbf{x}_{B}, \mathbf{x}_{A}\right)$. We evaluate this inversion in the time domain for each location $\mathbf{x}_{B}$ individually, where the integration path $\Omega$ is limited to a small window around the receiver location right above $\mathbf{x}_{B}$. In the example below, this window was chosen nine traces wide only. This is a strongly overdetermined problem that can be solved by least-squares inversion without additional regularization.

\section{WAVEFIELD SEPARATION}

We assume that horizontal receiver arrays are available at multiple depth levels. We define two unknowns: the downgoing field $\hat{\mathbf{V}}_{A}^{+}$and the upgoing field $\hat{\mathbf{V}}_{A}^{-}$, both evaluated at level $z_{A}$. These fields are expressed here as monochromatic matrices, following the notation of Berkhout (1993). In this notation, columns represent source locations and rows represent receiver 


\section{Wavefield separation using sensors at multiple depths on land}

locations. By the procedure described above, the propagators between level $z_{A}$ and another level $z_{B}$ can be estimated. These propagators are expressed as $\hat{\mathbf{W}}_{B A}$ for forward extrapolation of the downgoing field and $\hat{\mathbf{W}}_{B A}^{*}$ for inverse extrapolation of the upgoing field, where we used equation 4 . At each depth level $z_{N}$ (for instance $\mathrm{N}=\mathrm{A}$ or $\mathrm{N}=\mathrm{B}$ ) the following equation should hold:

$$
\hat{\mathbf{V}}_{N}=\hat{\mathbf{V}}_{N}^{+}+\hat{\mathbf{V}}_{N}^{-}
$$

Equations 1 and 3 can be written compactly as $\hat{\mathbf{V}}_{N}^{+}=\hat{\mathbf{W}}_{N A} \hat{\mathbf{V}}_{A}^{+}$ and $\hat{\mathbf{V}}_{N}^{-}=\hat{\mathbf{W}}_{N A}^{*} \hat{\mathbf{V}}_{A}^{-}$. Substituting these representations into equation 12 yields

$$
\hat{\mathbf{V}}_{N}=\hat{\mathbf{W}}_{N A} \hat{\mathbf{V}}_{A}^{+}+\hat{\mathbf{W}}_{N A}^{*} \hat{\mathbf{V}}_{A}^{-}
$$

Since we have measured the particle velocity field at multiple levels, we can construct multiple matrix equations depending on 2 unknown matrices. This leads to a relatively straightforward inverse problem for the upgoing and downgoing fields at level $z_{A}$. To solve this problem, we write equation 13 in the following way:

$$
\left(\begin{array}{c}
\hat{\mathbf{V}}_{A} \\
\hat{\mathbf{V}}_{B} \\
\hat{\mathbf{V}}_{C} \\
\ldots
\end{array}\right)=\hat{\mathbf{S}}\left(\begin{array}{c}
\hat{\mathbf{V}}_{A}^{+} \\
\hat{\mathbf{V}}_{A}^{-}
\end{array}\right),
$$

where the system matrix $\hat{\mathbf{S}}$ is given by

$$
\hat{\mathbf{S}}=\left(\begin{array}{cc}
\hat{\mathbf{W}}_{A A} & \hat{\mathbf{W}}_{A A}^{*} \\
\hat{\mathbf{W}}_{B A} & \hat{\mathbf{W}}_{B A}^{*} \\
\hat{\mathbf{W}}_{C A} & \hat{\mathbf{W}}_{C A}^{*} \\
\ldots & \ldots
\end{array}\right)
$$

Note that $\hat{\mathbf{W}}_{A A}=\hat{\mathbf{W}}_{A A}^{*}=\mathbf{I}$, being an identity matrix. Equation 14 will be solved by regularized least-squares inversion to provide the upgoing and downgoing field at the upper receiver level. This can be done using the recordings at two receiver levels $z_{A}$ and $z_{B}$ (being an even-determined inverse problem) or more than two receiver levels (being an overdetermined inverse problem).

\section{EXAMPLE}

The following synthetic example is inspired by recent experiment over an onshore field in Saudi Arabia that is described in more detail by Bakulin et al. (2012) and Alexandrov et al. (2012). A total of 641 sources are placed at the surface with a dense $7.5 \mathrm{~m}$ spacing. Now, 81 receivers are deployed in two arrays that are located at $60 \mathrm{~m}$ and $70 \mathrm{~m}$ below the surface. The target reservoir is located much deeper, at $2000 \mathrm{~m}$. The horizontal receiver spacing is $30 \mathrm{~m}$. To avoid spatial aliasing, we applied a Synthetic-Aperture-Source (SAS) filter (van der Neut, 2012), damping the high wavenumbers. This filter is also imposed on the unknown upgoing and downgoing fields that will be retrieved by wavefield separation. Later on in our processing sequence, we aim to deconvolve the upgoing field with the downgoing field for free-surface multiple elimination (van der Neut, 2012). During this processing step, the imprint of the filter will be removed again. In Figure 2 we show parts of the common-source gathers of the input data at $z_{A}$ and $z_{B}$, containing the target reflection of the reservoir, which is hard to identify due to severe multiple reflections. To estimate the propagator for wavefield extrapolation, we windowed the direct fields and computed the point-spread function and correlation function with equations 7 and 8 . Common-receiver gathers of these functions are shown in Figure 3. Time-shifts $\Delta t_{W}\left(\mathbf{x}_{B}, \mathbf{x}_{A}\right)$ are picked from the correlation function and amplitudes $A_{W}\left(\mathbf{x}_{B}, \mathbf{x}_{A}\right)$ are computed by inversion of equation 11 . Next, the propagator is synthesized with equation 9. In Figure 4a we show a common-receiver gather of the result. In Figure $4 \mathrm{~b}$ we show that forward-propagating the point-spread function with this propagator yields indeed a good estimate of the correlation function that we showed in Figure $3 b$.
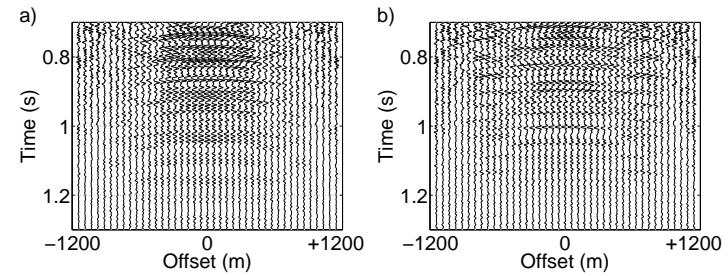

Figure 2: Common-source gather of the particle velocity field after SAS-filtering at a) level $z_{A}(60 \mathrm{~m})$ and b) level $z_{B}(70 \mathrm{~m})$.
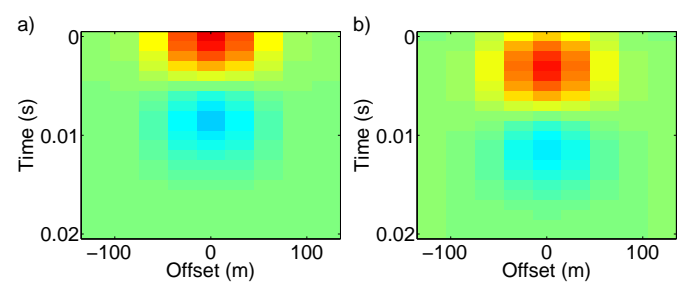

Figure 3: a) Common-receiver gather of the point-spread function and b) correlation function.

We applied wavefield separation to the input data by inverting the scheme as presented in equations 14 and 15. Results are shown in Figure 5, overlying the result of summing pressure and particle velocity fields at level $z_{A}$ as proposed by Mehta et al. (2007). A close match is observed. The decomposed fields could well be used for further processing. One option is to apply interferometry by multidimensional deconvolution (Wapenaar et al., 2011). In this method, the upgoing field is temporally and spatially deconvolved by the downgoing field. This process results in a data set as if sources and receiver are located at the receiver level with the overburden replaced by a homogeneous halfspace. In Figure 6a we show a reference response for this case, obtained by direct modeling. In Figure $6 \mathrm{~b}$ we show the retrieved result. In both responses the target reflector can clearly be observed at $t \approx 1.05 \mathrm{~s}$. 


\section{Wavefield separation using sensors at multiple depths on land}

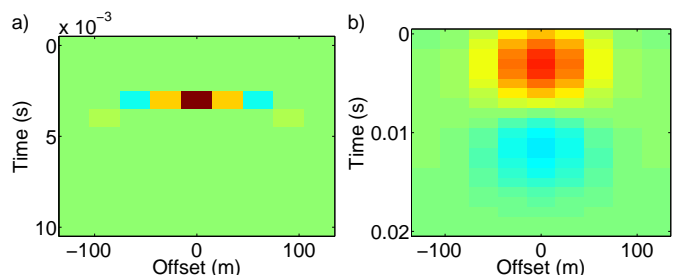

Figure 4: a) Common-receiver gather of the estimated propagator. b) Common-receiver gather of the estimated correlation function that is obtained by forward propagating the pointspread function. Note the close similarity with Figure $3 \mathrm{~b}$.
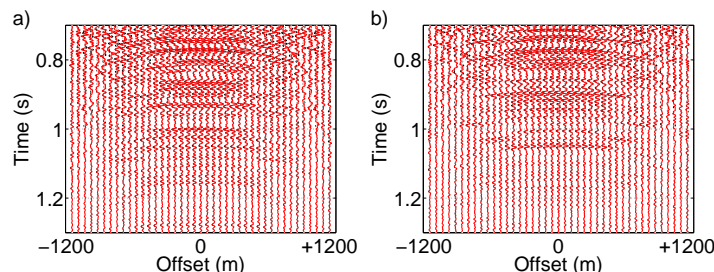

Figure 5: a) Downgoing and b) upgoing vertical particle velocity field retrieved by PV-summation (black) and dual-arraydecomposition (red). Every second trace is shown.

\section{DISCUSSION}

The success of this method depends on the invertibility of matrix $\hat{\mathbf{S}}$ as given by equation 15 . The invertibility depends strongly on the vertical separation of the receiver array elements and the data bandwidth. This is easiest to demonstrate for vertical wave propagation. In this case we can limit the aperture $\Omega_{A}$, over which equation 14 is evaluated, to a single trace. The system matrix from equation 15 can now be written as

$$
\hat{\mathbf{S}}=\left(\begin{array}{cc}
1 & 1 \\
A_{W} \exp \left(-j \omega \Delta t_{B A}\right) & A_{W} \exp \left(j \omega \Delta t_{B A}\right) \\
\ldots & \ldots
\end{array}\right)
$$

where $\Delta t_{B A}$ is the one-way traveltime between the receiver arrays $\mathrm{A}$ and $\mathrm{B}$. Assuming $\bar{c}_{B A}$ to be the average velocity array $\mathrm{A}$ and $\mathrm{B}$, it follows that $\Delta t_{B A}=\Delta z_{B A} / \bar{c}_{B A}$, where $\Delta z_{B A}$ is the spacing between the arrays. In the example, $\Delta t_{B A} \approx 3 \mathrm{~ms}$, which can also be observed from Figure $4 a$. In Figure $7 \mathrm{a}$ we show the condition number of matrix $\hat{\mathbf{S}}$. The condition number is the ratio of the largest and smallest singular value of the matrix. High condition numbers indicates that the matrix is poorly conditioned and hard to invert. We clearly observe a peak at $f_{c}=167 \mathrm{~Hz}$. To understand this peak, we evaluate the argument of the exponents in equation 16 , being $-j 2 \pi f_{c} \Delta t_{B A}=$ $-j \pi$ and $j 2 \pi f_{c} \Delta t_{B A}=j \pi$. Since $\exp (-j \pi)=\exp (j \pi)=-1$, the rows of matrix $\hat{\mathbf{S}}$ are no longer linearly independent and the matrix becomes uninvertible. This so-called notch frequency $f_{c}$ depends on $\Delta t_{B A}$ and therefore directly on the average velocity $\bar{c}_{B A}$ and spacing $\Delta z_{B A}$. In Figure $7 \mathrm{a}$ we show the condition numbers of $\hat{\mathbf{S}}$ for another array with $\Delta z_{B A}=20 \mathrm{~m}$, giving a notch frequency of $83 \mathrm{~Hz}$. In this case the notch fre- quency overlaps with the bandwidth of the data, such that we can no longer invert the problem without additional notch filtering. The frequency notch also depends on the wave velocity $\bar{c}_{B A}$. In figure $7 \mathrm{~b}$ we show the condition numbers for a lower wave velocity $\bar{c}_{B A}=2000 \mathrm{~m} / \mathrm{s}$ with $\Delta z_{B A}=10 \mathrm{~m}$, causing the notch frequency to occur at $100 \mathrm{~Hz}$. To circumvent the notch, we can make use of multiple receiver arrays but they should be unequally spaced or have different interval wave velocities. As an example we computed the response of another array $\mathrm{C}$ with $\Delta z_{C A}=25 \mathrm{~m}$ in Figure $7 \mathrm{~b}$, using the same velocity $\bar{c}_{C A}=\bar{c}_{B A}=2000 \mathrm{~m} / \mathrm{s}$. A number of notches can be observed, however not at $100 \mathrm{~Hz}$. Therefore, inversion with all three arrays should be well possible, as can be seen from the condition numbers of the combined problem in Figure $7 b$.
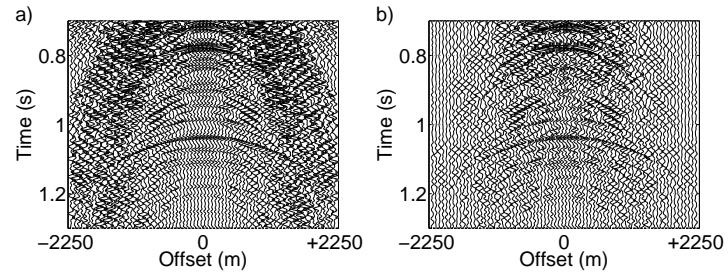

Figure 6: a) Reference response from direct modeling and b) retrieved response of a virtual source and receivers at $z_{A}=60 \mathrm{~m}$ in a medium that is homogeneous above $z_{A}$. Every second trace is shown. In both responses an average has been taken over common offsets.
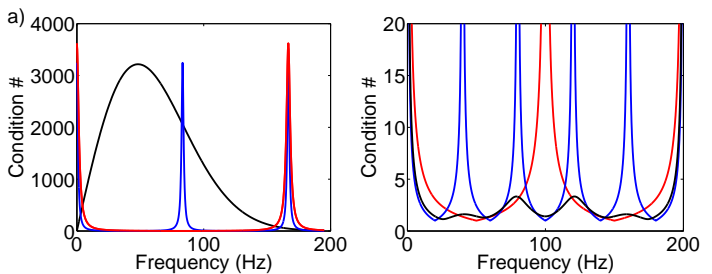

Figure 7: a) Condition numbers with $\Delta z_{B A}=10 \mathrm{~m}$ (red) and $\Delta z_{B A}=20 \mathrm{~m}$ (blue) (both with $\bar{c}_{B A}=3000 \mathrm{~m} / \mathrm{s}$ ). In black we show the frequency content of the data. b) Condition numbers with $\bar{c}_{B A}=\bar{c}_{C A}=2000 \mathrm{~m} / \mathrm{s}$ with $\Delta z_{B A}=10 \mathrm{~m}$ (red) and $\Delta z_{C A}=$ $25 m$ (blue). In black we show the condition numbers for an inversion problem that utilizes all three arrays A, B and C.

\section{CONCLUSION}

We have presented an inversion scheme for separation of upgoing and downgoing wavefields using receiver arrays at multiple depth levels. The invertibility of the system depends on the vertical spacing between the receiver arrays. For vertical propagation and a fixed frequency, the worst sensor spacing is half of the wavelength, whereas optimum spacing is a quarter of the wavelength. In this case, an approximation of this methodology can also be applied with two (or more) vertically spaced sensors in a single vertical hole. For non-vertical propagation, the worst and optimum spacings will be slightly smaller and multiple receivers should be deployed at each depth level. 


\section{Wavefield separation using sensors at multiple depths on land}

\section{REFERENCES}

Alexandrov, D., A. Bakulin, and R. Burnstad, 2012, Virtual source redatuming of synthetic land data acquired with shallow buried receivers: 74th EAGE Conference and Exhibition, Expanded Abstracts, P252.

Bakulin, A., R. Burnstad, M. Jervis, and P. Kelamis, 2012, The feasibility of permanent land seismic monitoring with buried geophones and hydrophones in a desert environment: 74th EAGE Conference and Exhibition, Expanded Abstracts, X038.

Bakulin, A. and R. Calvert, 2006, The virtual source method: Theory and case study: Geophysics, 71, SI139-SI150.

Berkhout, A. J., 1993, A unified approach to acoustical reflection imaging: Journal of the Acoustical Society of America, 93, 2005-2017.

Burnstad, R., A. Bakulin, M. Jervis, and D. Alexandrov, 2012a, Successful imaging of land hydrophone and dual sensor data in a dry desert environment: 82nd Annual Meeting, SEG, Expanded Abstracts, 1-5.

Burnstad, R., M. Jervis, and A. Bakulin, 2012b, A novel method of 4d analysis and its application to land seismic data: 74th EAGE Conference and Exhibition, Expanded Abstracts, E012.

Claerbout, J. F., 1971, Toward a unified theory of reflector mapping: Geophysics, 36, 467-481.

Kluver, T., P. Aaron, D. Carlson, A. Day, and R. van Borselen, 2009, A robust strategy for processing 3d dual-sensor towed streamer data: 79th Annual Meeting, SEG, Expanded Abstracts, 3556-3560.

Loewenthal, D. and E. A. Robinson, 2000, On unified dual fields and einstein deconvolution: Geophysics, 65, $293-303$.

Mehta, K., R. Bakulin, A. Snieder, J. Sheiman, and R. Calvert, 2007, Improving the virtual source method by wavefield separation: Geophysics, 72, V79-V86.

Mehta, K., D. Kiyashchenko, P. Jorgensen, J. Lopez, J. Ferrandis, and M. Costello, 2010, Virtual source method applied to crosswell and horizontal well geometries: The Leading Edge, 29, 712-723.

Petronio, L. and F. Poletto, 2010, Dual signals separation in shallow borehole hydrophone data: 72nd EAGE Conference and Exhibition, Expanded Abstracts, F033.

Posthumus, B. J., 1993, Deghosting using a twin streamer configuration: Geophysical Prospecting, 41, 267-286.

Schalkwijk, K. M., C. P. A. Wapenaar, and D. J. Verschuur, 1999, Application of two-step decomposition to multicomponent ocean-bottom data: theory and case study: Journal of Seismic Exploration, 8, 261-278.

van der Neut, J., 2012, Interferometric redatuming by multidimensional deconvolution: PhD dissertation thesis, Delft University of Technology.

Wapenaar, K., 1998, Reciproticy properties of one-way propagators: Geophysics, 63, 1795-1798.

Wapenaar, K., J. van der Neut, E. Ruigrok, D. Draganov, J. Hunziker, E. Slob, J. Thorbecke, and R. Snieder, 2011, Seismic interferometry by crosscorrelation and by multi-dimensional deconvolution: a systematic comparison: Geophysical Journal International, 185, 1335-1364. 
http://dx.doi.org/10.1190/segam2013-0410.1

\section{EDITED REFERENCES}

Note: This reference list is a copy-edited version of the reference list submitted by the author. Reference lists for the 2013 SEG Technical Program Expanded Abstracts have been copy edited so that references provided with the online metadata for each paper will achieve a high degree of linking to cited sources that appear on the Web.

\section{REFERENCES}

Alexandrov, D., A. Bakulin, and R. Burnstad, 2012, Virtual source redatuming of synthetic land data acquired with shallow buried receivers: 74th Conference \& Exhibition, EAGE, Extended Abstracts, P252.

Bakulin, A., R. Burnstad, M. Jervis, and P. Kelamis, 2012, The feasibility of permanent land seismic monitoring with buried geophones and hydrophones in a desert environment: 74th Conference \& Exhibition, EAGE, Extended Abstracts, X038.

Bakulin, A., and R. Calvert, 2006, The virtual source method: Theory and case study: Geophysics, 71, no. 4, SI139-SI150, http://dx.doi.org/10.1190/1.2216190.

Berkhout, A. J., 1993, A unified approach to acoustical reflection imaging. 1: The forward model: Journal of the Acoustical Society of America, 93, 2005-2016, http://dx.doi.org/10.1121/1.406714.

Burnstad, R., A. Bakulin, M. Jervis, and D. Alexandrov, 2012a, Successful imaging of land hydrophone and dual sensor data in a dry desert environment: 82nd Annual International Meeting, SEG, Expanded Abstracts, doi:10.1190/segam2012-0957.1.

Burnstad, R., M. Jervis, and A. Bakulin, 2012b, A novel method of 4D analysis and its application to land seismic data: 74th Conference \& Exhibition, EAGE, Extended Abstracts, E012.

Claerbout, J. F., 1971, Toward a unified theory of reflector mapping: Geophysics, 36, 467-481, http://dx.doi.org/10.1190/1.1440185.

Kluver, T., P. Aaron, D. Carlson, A. Day, and R. van Borselen, 2009, A robust strategy for processing 3D dual-sensor towed streamer data: 79th Annual International Meeting, SEG, Expanded Abstracts, 3088-3092.

Loewenthal, D., and E. A. Robinson, 2000, On unified dual fields and Einstein deconvolution: Geophysics, 65, 293-303, http://dx.doi.org/10.1190/1.1444720.

Mehta, K., R. Bakulin, J. Sheiman, R. Calvert, and A. Snieder, 2007, Improving the virtual source method by wavefield separation: Geophysics, 72, no. 4, V79-V86, http://dx.doi.org/10.1190/1.2733020.

Mehta, K., D. Kiyashchenko, P. Jorgensen, J. Lopez, J. Ferrandis, and M. Costello, 2010, Virtual source method applied to crosswell and horizontal well geometries: The Leading Edge, 29, 712-723, http://dx.doi.org/10.1190/1.3447785.

Petronio, L., and F. Poletto, 2010, Dual signals separation in shallow borehole hydrophone data: 72nd Conference \& Exhibition, EAGE, Extended Abstracts, F033.

Posthumus, B. J., 1993, Deghosting using a twin streamer configuration: Geophysical Prospecting, 41, no. 3, 267-286, http://dx.doi.org/10.1111/j.1365-2478.1993.tb00570.x.

Schalkw ijk, K. M., C. P. A. Wapenaar, and D. J. Verschuur, 1999, Application of two-step decomposition to multicomponent ocean-bottom data: Theory and case study: Journal of Seismic Exploration, 8, 261-278. 
van der Neut, J., 2012, Interferometric redatuming by multidimensional deconvolution: Ph.D. dissertation, Delft University of Technology.

Wapenaar, K., 1998, Reciprocity properties of one-way propagators: Geophysics, 63, 1795-1798, http://dx.doi.org/10.1190/1.1444473.

Wapenaar, K., J. van der Neut, E. Ruigrok, D. Draganov, J. Hunziker, E. Slob, J. Thorbecke, and R. Snieder, 2011, Seismic interferometry by crosscorrelation and by multidimensional deconvolution: A systematic comparison: Geophysical Journal International, 185, 1335-1364, http://dx.doi.org/10.1111/j.1365-246X.2011.05007.x. 